\title{
After fire, the roles of rabbits and wildflowers
}

\author{
The dark ash left by a chaparral fire is rich in ammonium nitrogen; can the ecosystem absorb it \\ before winter rains wash it away?
}

Herbaceous annual plants can act as sponges for nitrogen.

Whisperingbells (Emmenanthe penduliflora) may cycle the nitrogen in wildfire ash faster than nitrogen-fixing herbaceous plants.

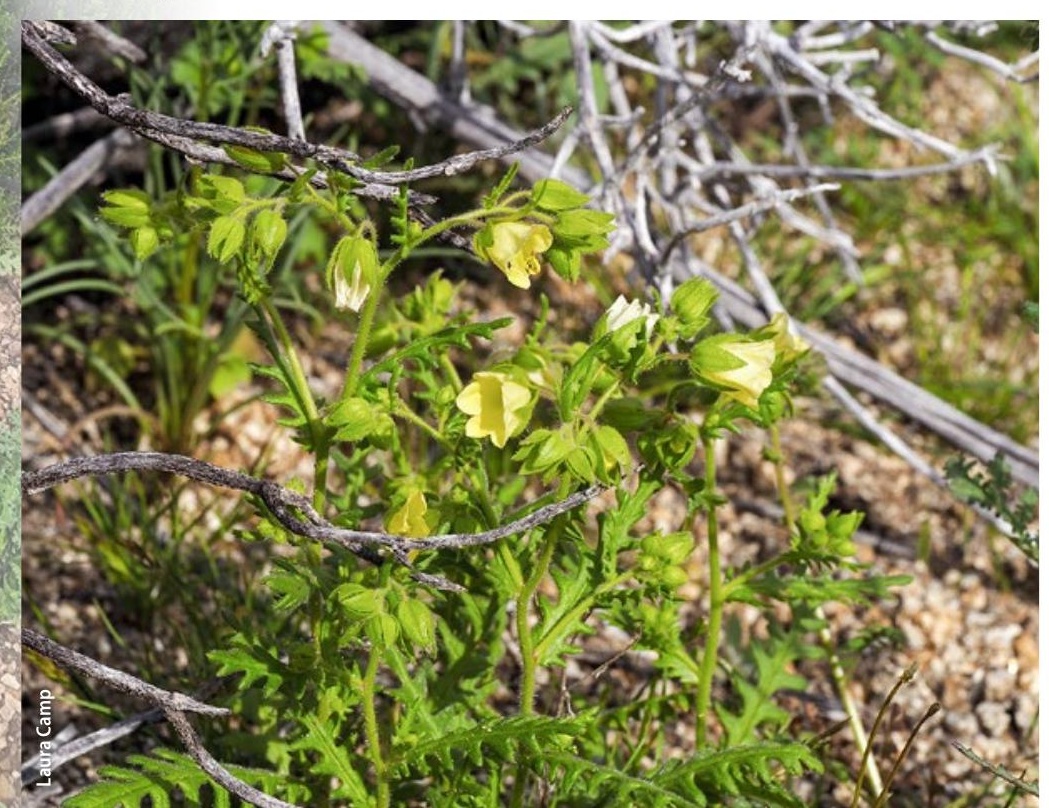
and grow quickly, the ash, and the nonabsorbed nitrogen, washes away."

Last year, Hendricks-Franco set fires at HREC in April and October, sampling for nitrogen before and after the fires. In mid-December, annuals were sprouting, and the chaparral shrubs burned in the spring fire showed some new growth. But so much rain had fallen, ash was also washing away.

In late December, fences were up around some of the burned plots, to study the effect of excluding herbivores. In January, HendricksFranco was weeding the plots so that they contained four different plant groups: (1) all naturally occurring herbaceous plants, (2) only plants that aren't nitrogen fixers, (3) only nitrogen-fixing plants and (4) no herbaceous plants at all.

She hypothesizes that the first group will cycle nitrogen most vigorously, followed by the

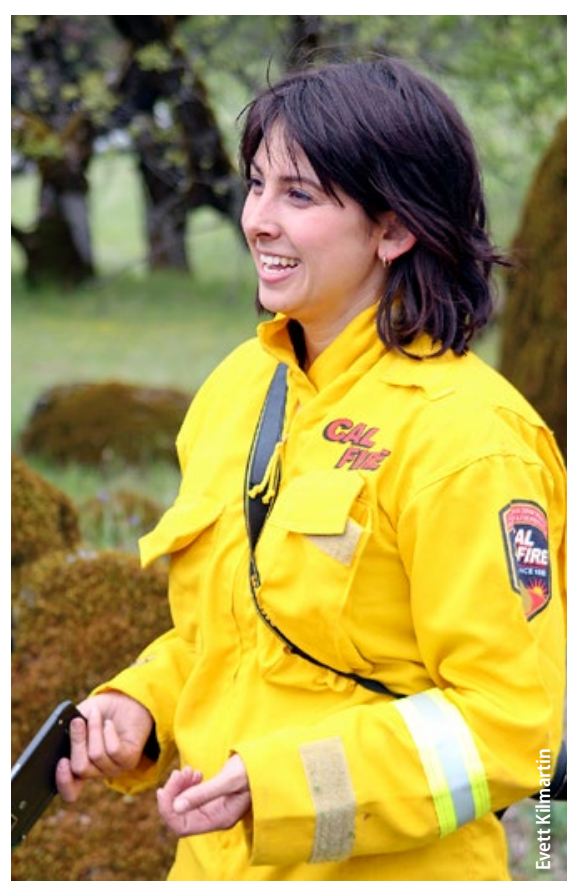

Reseacher Lindsey HendricksFranco hypothesizes that herbivores will accelerate rich chaparral systems. nitrogen cycling in nitrogensecond, third and fourth, in that order. Additionally, she thinks herbivores will accelerate nitrogen cycling in some plots, because in high-nitrogen systems, they stimulate growth of fast-growing plants that can tolerate grazing. Nitrogen sampling during the next two years will reveal how well different plant communities retain nitrogen after fire, with and without herbivores, and the effect of fire season.

This work requires a trial in a natural setting such as HREC, which has mature chaparral that is accessible and can be burned safely. Until now, there had been no formal replicated fire trials with control plots to measure the effects of animal grazing on nitrogen cycling after chaparral fire. The study results will help guide land managers on what to do in the first few months after a fire - perhaps seeding native plants and introducing grazers - to encourage fast recovery of the chaparral and associated species. CA 